\title{
Effects of thermoultrasonic treatment on characteristics of micro-nano particles and flavor in Greenland halibut bone soup
}

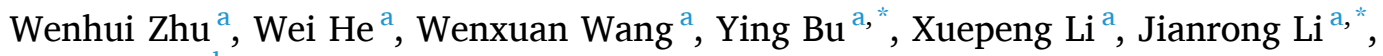 \\ Yuyu Zhang ${ }^{\mathrm{b}}$ \\ ${ }^{a}$ College of Food Science and Engineering, Bohai University. Jinzhou, Liaoning 121013, China \\ ${ }^{\mathrm{b}}$ Beijing Key Laboratory of Flavor Chemistry, Beijing Technology and Business University (BTBU), Beijing 100048, China
}

\section{A R T I C L E I N F O}

\section{Keywords:}

Halibut bone soup

Thermoultrasonic treatment (TUT)

Micro-nano particles (MNPs)

Flavor

Headspace-gas chromatography-ion mobility

spectrometry (HS-GC-IMS)

\begin{abstract}
A B S T R A C T
In order to investigate the effects of thermoultrasonic treatment (TUT) on the formation of colloidal micro-nano particles (MNPs) and the quality of halibut bone soup, nutrients, particle characteristics, and flavor characteristics were analyzed. The morphology of MNPs was studied using an optical microscope. Results showed that TUT could increase the nutrient content (total sugars, $22.15 \mathrm{mg} / 100 \mathrm{~mL}$; water soluble proteins, $173.24 \mathrm{mg} / \mathrm{mL}$; fatty acids, $1779.7 \mathrm{mg} / 100 \mathrm{~mL}$; solids, $3.16 \mathrm{~g} / 100 \mathrm{~mL})$, reduce the particle size $(605.92 \mathrm{~nm})$ and interfacial tension. Meanwhile, TUT make the halibut bone soup has better emulsifying characteristics and stability. The contents of flavor substances, such as esters, $5^{\prime}$-nucleotides, organic acids in the halibut bone soup were more abundant, while the contents of hexanal and 1-octen-3-ol and fishy off-flavor were reduced in TUT group. The overall odor and taste outline were more harmonious. Therefore, TUT can be used in the production of high quality fish bone soup, and TUT could be considered as a good deep processing technology for halibut bone and improve economic efficiency.
\end{abstract}

\section{Introduction}

Soup is one of the easy-to-prepare and nutritional food items and are thus an indispensable part of the world food culture, such as Chinese soup, gazpacho, borscht, etc. Soup can be regarded as a complex colloid system, and the making process of soup complies with diffusion theory and Fick's law [1]. Due to the continuous heating process, nutrients and flavor components in soup migrate and diffuse as chemical bonds and interactions are broken and generated [2]. These components dissolve and diffuse in the soup and generate new aggregation and assembly through a series of physical and chemical reactions. The new aggregates formed are generally micron or nanometer scale, which is called micronano particles (MNPs). MNPs impart sensory, flavor, functional and nutritional properties to soups, resulting in unique physical and chemical properties. At the same time, compared with chemically synthesized MNPs, food-derived MNPs have surprisingly good biocompatibility, functionality, stability and lower toxicity [3]. The existing research is to interpret the formation mechanism through the simple aggregation of substances and the interaction between single molecules $[4,5]$.

As a promising non-thermal processing technology, ultrasonic processing has great potential to control, improve and accelerate the process without compromising the quality of food and other products [6]. Ultrasonic processing devices use piezoelectric materials to convert electrical energy into mechanical energy, which produces ultrasonic waves [7]. Ultrasonic processing waves alternately produce high pressure and low pressure in the medium, resulting in compression and expansion cycles of the medium. The cavitation bubble is created during the cycle and grows as the cycle progresses until it can no longer absorb any more energy. The cavitation bubbles then collapse violently and release energy, forming regions of high temperature and pressure, a phenomenon known as cavitation [8]. Cavitation can destroy the substrate of food raw materials, and promote the release of substances in the substrate as well as the material exchange and reaction between the substrate and the medium [9]. Ultrasonic processing combined with heat (temperature $\geq 50{ }^{\circ} \mathrm{C}$ ) is called thermoultrasonic [10]. Thermoultrasonic can effectively improve food processing technology by using cavitation and heat. And it has a wide range of applications in extraction, emulsification, sterilization, reaction kinetics and crystallization technology $[11,12]$. At present, the effect of thermoultrasonic treatment (TUT) on MNPs in soup has not been reported.

\footnotetext{
* Corresponding authors at: College of Food Science and Engineering, Bohai University, No. 19, Keji Road, Jinzhou 121013, China.

E-mail addresses: buying@bhu.edu.cn (Y. Bu), lijianrong@bhu.edu.cn (J. Li).
} 
Greenland halibut (Reinhardtius hippoglossoides) is one of the most economically valuable flatfishes of the North Atlantic region, and is rich in protein, mineral elements and unsaturated fatty acids. Because of its tender meat, it is often processed into frozen fish fillets and hot smoked fish fillets. The loss rate can reach $65 \%$ in processing [13], resulting in a huge waste. The skeleton is the main by-product in the halibut processing process, accounting for about $15 \%$ of the weight of the fish [14], and contain a lot of meat between their bones. Due to the high fat content of halibut (10\%) [15], halibut skeletons can be prepared into thick soup by non-frying process. As consumers worldwide are increasingly concerned about health issues, the demand for a low fat, non-fried processing food has increased rapidly. Herein, the non-fried halibut fish bone soup which make the processing procedure easier, saving processing time just cater to this development.

In this study, the effects of TUT on the nutrients in halibut bone soup, MNPs characteristics and system characteristics, and the effects of ultrasound on the flavor profile of halibut bone soup were studied. The results of this study can provide a rapid and practical means for improving the nutritional value and flavor evaluation of halibut bone soup, and improving the stability of the bone soup system.

\section{Materials and methods}

\subsection{Materials and reagents}

Frozen Greenland halibut bones were purchased from Shandong Meijia Group Co. Ltd (Shandong, China) and stored at $-20{ }^{\circ} \mathrm{C}$. Succinic acid, lactic acid malic acid were purchased from Solibol Technology Co., Ltd. (Beijing, China). Methanol (chromatographic grade) was purchased from Sigma Co., Ltd. (Shanghai, China). Inosine-5'-monphosphate (IMP), Adenosine-5'-monophosphate (AMP) and Guanosine-5'-monophosphate (GMP) standards were purchased from Sigma Co., Ltd. (Shanghai, China). All the chemicals used in different analyses were of analytical grade, unless otherwise mentioned.

\subsection{Preparation of halibut bone soup}

The halibut bone was washed and cut into pieces (about $3-5 \mathrm{~cm}$ ), then smashed into bone mud. According to the preliminary experiment in the laboratory, the fish bone soup boiling conditions are the ratio of bone mud to water $1: 6,100{ }^{\circ} \mathrm{C}$ boiling for $150 \mathrm{~min}$. Specifically, $100 \mathrm{~g}$ bone mud and $600 \mathrm{~mL}$ deionized water were packed separately in high temperature cooking bags. Ultrasonic treatment was performed with an ultrasound-assisted quick-freezing device (XO-120L-II, Xianou Co., Ltd., Nanjing, China), and the ultrasonic conditions were $25 \mathrm{kHz}, 400 \mathrm{~W}$, and $10 \mathrm{~min}$. The samples were filtered by a double-layer 200 mesh gauze and stored separately at $-80^{\circ} \mathrm{C}$.

\subsection{Determination of nutrient content}

\subsubsection{Total sugar}

The total sugar content was determined by the sulfuric acid-anthrone method using an ultraviolet spectrophotometer (UV-2550, Shimadzu Instruments Co., Ltd.), referring to the method of Leng et al. [16].

\subsubsection{Water-soluble proteins}

The content of water-soluble protein in the sample was determined by biuret calorimetry. Referring to the method of Subroto et al. [17] with a slight modification, the diluted sample was mixed with $4 \mathrm{~mL}$ biuret reagent, thoroughly mixed, and placed at $20-25{ }^{\circ} \mathrm{C}$ for $30 \mathrm{~min}$. The absorbance was measured at $540 \mathrm{~nm}$. With 0, 0.25, 0.5, 0.75, 1 and $1.25 \mathrm{mg} / \mathrm{mL}$ bovine serum albumin as the standard samples. Linear regression equation was used to calculate the content of water-soluble protein.

\subsubsection{Fatty acids}

The fatty acids content was determined referring to the method of Zcan et al. [18] with some modifications. Sample $(0.1 \mathrm{~mL})$ and C19:0 internal standard $(100 \mu \mathrm{L}, 10 \mathrm{~mol} / \mathrm{mL})$ were mixed with methanolsodium hydroxide ( $5 \mathrm{~mL}, 0.5 \mathrm{~mol} / \mathrm{mL}$ ), concentrated at $100{ }^{\circ} \mathrm{C}$ for 10 min. Subsequently, boron trifluoride methanol ( $3 \mathrm{~mL}, 14 \%$ methanol), $\mathrm{n}$-hexane $(2 \mathrm{~mL})$ and saturated sodium chloride solution $(10 \mathrm{~mL})$ were added to the mixed solution. The mixture was centrifuged at $6000 \mathrm{~g}$ for 5 min. After centrifugation, the upper sample was injected into a gas chromatograph (7890A, Agilent Technologies Co Ltd, USA) for fatty acids analysis.

\subsubsection{Solids}

The solids content of the sample was determined in an oven at $102-105^{\circ} \mathrm{C}$ until it was dried to a constant weight.

\subsection{Color analysis}

The difference in chromaticity $L, a^{*}, b^{*}$ between the samples was measured with a color meter (CR-400, Konica Minolta, Japan). Where, $L$ stands for brightness; $a^{*}$ stands for red-greenness, $b$ * stands for yellowblueness.

\subsection{Particle size, emulsion properties analysis and appearance structure observation of MNPs in halibut bone soup}

\subsubsection{Particle size property analysis}

The particle size was measured using a Zeta potentiometer (NanoBrook 90 Plus Zeta, Brookhaven Instrument Co., Ltd., USA). Distilled water was used as the diluent, the samples were diluted 10 times. The mean particle diameter and distribution coefficient of the samples were recorded with the matching Zetasizer software. The measurements were repeated three times for each sample.

\subsubsection{Interfacial tension}

The interfacial tension was measured using the droplet shape analysis (DSA) method and was carried out on the OCA-20 instrument (German Data Physics Instrument Co., Ltd.). Referred to the method of Chen et al. [19] with a slight modification. A drop (10 $\mu \mathrm{L})$ of sample was injected into an optical quartz cuvette containing purified soybean oil and kept in a vertical position at the end of the capillary tip (diameter $1.19 \mathrm{~mm}$ ) at room temperature. A charge-coupled device (CCD) camera was used to continuously monitor the shape of the droplet, and the workstation software was used to automatically calculate the interfacial tension according to the basic Yong Laplace equation [20].

\subsubsection{Kinetic stability}

In order to study the shelf life of the samples in cold storage $\left(4^{\circ} \mathrm{C}\right)$, the kinetic stability of the samples was measured. The determination method was referred to Wang et al. [21] with some modifications. The sample $(10 \mathrm{~mL})$ was taken and placed in a test tube, sealed and stored at $4{ }^{\circ} \mathrm{C}$ for $24 \mathrm{~h}$, and the upper and lower separated phases were observed. The initial total height of the sample before storage was denoted as $\mathrm{H}_{\mathrm{T}}$. The height of the upper phase of the sample stored for $24 \mathrm{~h}$ was measured and recorded as $\mathrm{H}_{\mathrm{X}}$. The separation index (SI, \%) of the sample was calculated using Eq. (1):

$$
S I(\%)=\frac{H_{X}}{H_{T}} \times 100
$$

\subsubsection{Appearance structure}

The appearance of the sample was recorded by camera, and the microstructure was observed and photographed by optical microscope (Niko80i, Nikon Corporation, Tokyo, Japan). The sample (20 $\mu \mathrm{L})$ was placed on a glass slide and covered with a cover slip. The excess sample was absorbed with filter paper. The samples were slightly air-dried and 
fixed, observed under a $400 \times$ microscope, and photographed for recording.

\subsection{Flavor analysis}

\subsubsection{Electronic nose (e-nose) analysis}

Aromatic information and volatile compound data were obtained using a portable e-nose system (PEN3, Win Muster Airsense Analytics Inc., Germany). According to the method of Zhu et al. [22], the sample $(5 \mathrm{~mL})$ was poured into a centrifuge tube $(50 \mathrm{~mL})$ and sealed with 3 layers of plastic wrap. Then, the sample was balanced in headspace for $30 \mathrm{~min}$ in a $37^{\circ} \mathrm{C}$ water bath to facilitate odor detection with an electronic nose probe. After cleaning the probe with filtered air for $120 \mathrm{~s}$, set the baseline (via automatic zero setting) for $5 \mathrm{~s}$ before the next measurement. The detection time and cleaning time were both $120 \mathrm{~s}$, and the gas flow rate was $300 \mathrm{~mL} / \mathrm{min}$. The experiment was repeated three times for each sample.

\subsubsection{Headspace-gas chromatography-ion mobility spectrometry (HS-GC- IMS) analysis}

The flavor of halibut fish bone soup was analyzed and determined on an Agilent 490 gas chromatograph (Agilent Technologies, Palo Alto, CA, USA) equipped with an MXT- 5 column ( $15 \mathrm{~m}, 0.53 \mathrm{~mm}$ ) and an IMS instrument FlavourSpec ${ }^{\circledR}$. The instrument is equipped with an autosampler unit, which can be directly sampled from the headspace using a $1 \mathrm{~mL}$ air-tight heated syringe.

Referred to the analysis methods of Chen et al. [23] and Fan et al. [24] with slight modifications. The halibut bone soup $(2 \mathrm{~mL})$ was placed in a $20 \mathrm{~mL}$ glass headspace bottle. Subsequently, the sample was incubated at a constant temperature of $60{ }^{\circ} \mathrm{C}$ for $20 \mathrm{~min}$ at a speed of 500 $\mathrm{rpm}$. After incubation, $500 \mu \mathrm{L}$ of headspace was pumped through a syringe heated at $65^{\circ} \mathrm{C}$, and then injected into the injector automatically. The IMS temperature was set to $45{ }^{\circ} \mathrm{C}$, and the drift gas flow rate was $150 \mathrm{~mL} / \mathrm{min}$. The sample was injected into the chromatographic column via carrier gas $\left(\mathrm{N}_{2}\right)$, and the program flow rate was set to $2 \mathrm{~mL} / \mathrm{min}$ for 2 $\mathrm{min}, 10 \mathrm{~mL} / \mathrm{min}$ for $8 \mathrm{~min}$, and $100 \mathrm{~mL} / \mathrm{min}$ for $10 \mathrm{~min}$. Volatile compounds were identified by comparing the drift time of the standard in RI and GC-IMS database.

\subsection{3. $5^{\prime}$ - nucleotides and organic acids analysis}

The organic acids and $5^{\prime}$-nucleotides level were analyzed by a HPLC system (Agilent1100, Agilent Technologies, Santa Clara, USA), referring to the method of Kong et al. [25] and Xu et al. [26] respectively.

\subsubsection{Free amino acids (FAAs)}

According to the method of Akagündüz et al. [27], the amino acid analyzer (L-8900, Hitachi, Tokyo, Japan) was used to measure the FAAs.

\subsection{Statistical analysis}

Origin 9.0 and TBtools were used for mapping and analysis. Statistical analysis was performed by one-way analysis of variance (ANOVA) and Duncan's multiple range test at $P<0.05$ using SPSS 22.0 (SPSS Inc., Chicago, IL, USA). All measurements were performed in at least three independent experiments and results were expressed as mean \pm standard deviation (SD). For HS-GC-IMS, the instrumental analysis software includes LAV (Laboratory Analytical Viewer) and three plug-ins as well as GC $\times$ IMS Library Search, which can be used for sample analysis from different angles.

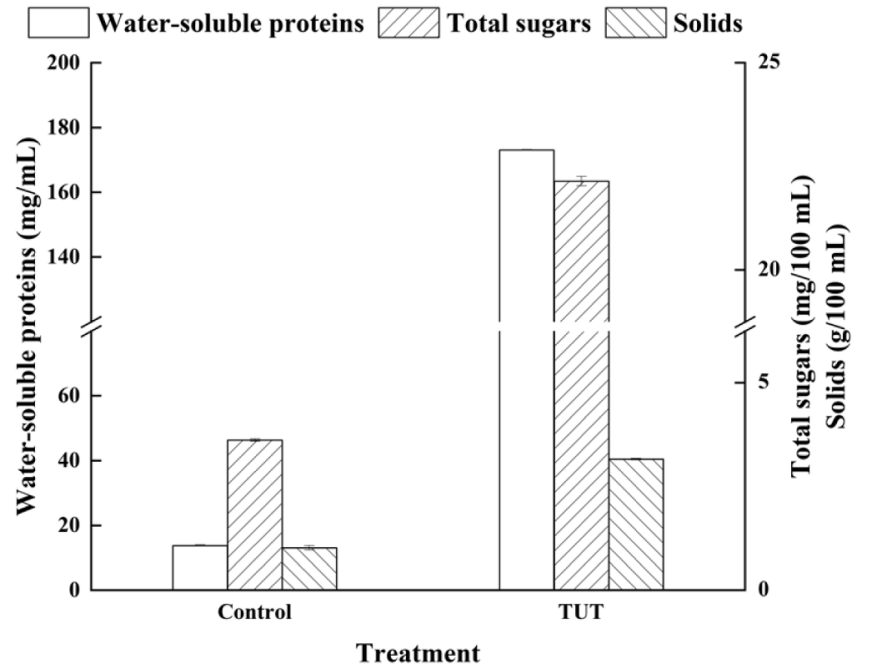

Fig. 1. Migration of total sugars, water-soluble proteins and solids in the halibut bone soup.

\section{Results and discussions}

\subsection{Effects of TUT on nutrient migration in halibut bone soup}

3.1.1. Effect of TUT on total sugars, water soluble proteins, and solids content in halibut bone soup

As indispensable nutrients in soup, sugars and proteins are important indicators for judging the quality of soup. The presence of high content of sugar and proteins will not only improve the nutritional value of the soup, but also give the soup rich flavor characteristics [28]. Solids can be used as a key indicator to measure the quality of fish bone soup, and it also reflects the overall dissolution of nutrients and flavor substances [29].

The standard curves of total sugars and proteins content were $\mathrm{y}=$ $0.1076 \mathrm{x}+0.0014\left(\mathrm{R}^{2}=0.9975\right)$ and $\mathrm{y}=0.0526 \mathrm{x}-0.0005\left(\mathrm{R}^{2}=0.9985\right)$, respectively. The effect of TUT on nutrient transport during the cooking process of halibut bone soup was shown in Fig. 1. TUT promoted the migration of nutrients from halibut bone mud to the soup, and greatly increased the contents of total sugars ( $3.62 \mathrm{~g} / 100 \mathrm{~mL}$ vs $22.15 \mathrm{~g} / 100$ $\mathrm{mL})$, water-soluble proteins $(13.78 \mathrm{mg} / \mathrm{mL}$ vs $173.11 \mathrm{mg} / \mathrm{mL})$ and solids $(1.02 \mathrm{~g} / 100 \mathrm{~mL}$ vs $3.16 \mathrm{~g} / 100 \mathrm{~mL})$ in the soup compared with the control group. This phenomenon could be explained by mechanical crushing and cavitation of TUT. The shock wave and shear force generated by these two effects could break the macromolecular nutrients in the bone mud, reducing the difficulty of dissolving the nutrients [30]. In addition, combined with the TUT in the boiling process, the broth could be given a relatively higher pressure and temperature [31], which promoted the migration and content balance of nutrients in the soup.

\subsubsection{Effect of TUT on fatty acids content in halibut bone soup}

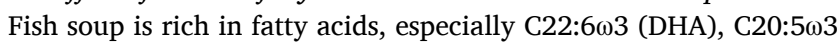
(EPA) and other $\omega-3$ polyunsaturated fatty acids (PUFA). These fatty acids play important roles in human health and nutrition. Because they can reduce the risk of coronary heart disease, prevent certain cancers, and improve immune function [32].

The fatty acids content of halibut bone soup in the control group included 8 kinds of fatty acids, including 2 kinds of saturated fatty acids (SFA), 5 kinds of monounsaturated fatty acids (MUFA) and 1 kind of polyunsaturated fatty acids (PUFA). In the TUT group, 14 kinds of fatty acids were detected, including 3 kinds of SFA, 5 kinds of MUFA and 6 kinds of PUFA. (Fig. 2 and Table S1). Higher levels of EPA and DHA were detected whereas none were detected in the control group. 
A

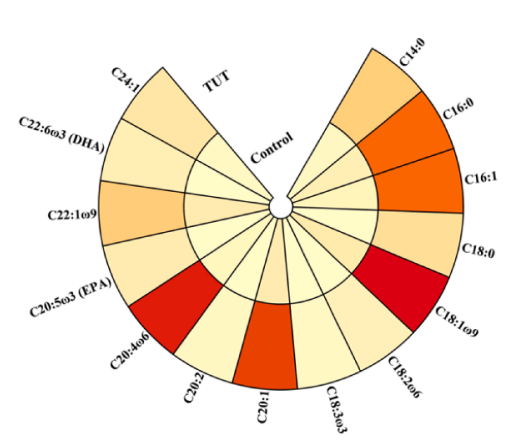

B

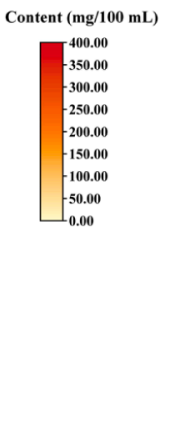

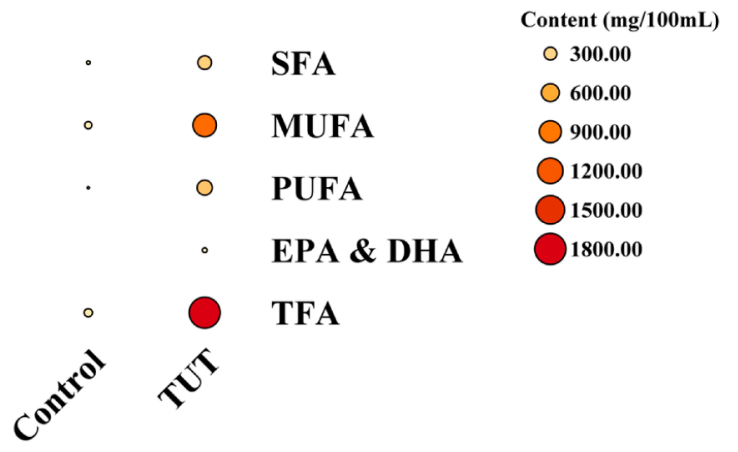

Fig. 2. Heat map of fatty acids content in the halibut bone soup.

Table 1

Changes of color in the halibut bone soup.

\begin{tabular}{llll}
\hline Treatment & $L$ & $a^{*}$ & $b^{*}$ \\
\hline Control & $38.47 \pm 0.21$ & $-0.37 \pm 0.03$ & $-2.77 \pm 0.14$ \\
TUT & $80.90 \pm 0.74$ & $-0.94 \pm 0.05$ & $0.85 \pm 0.29$ \\
\hline
\end{tabular}

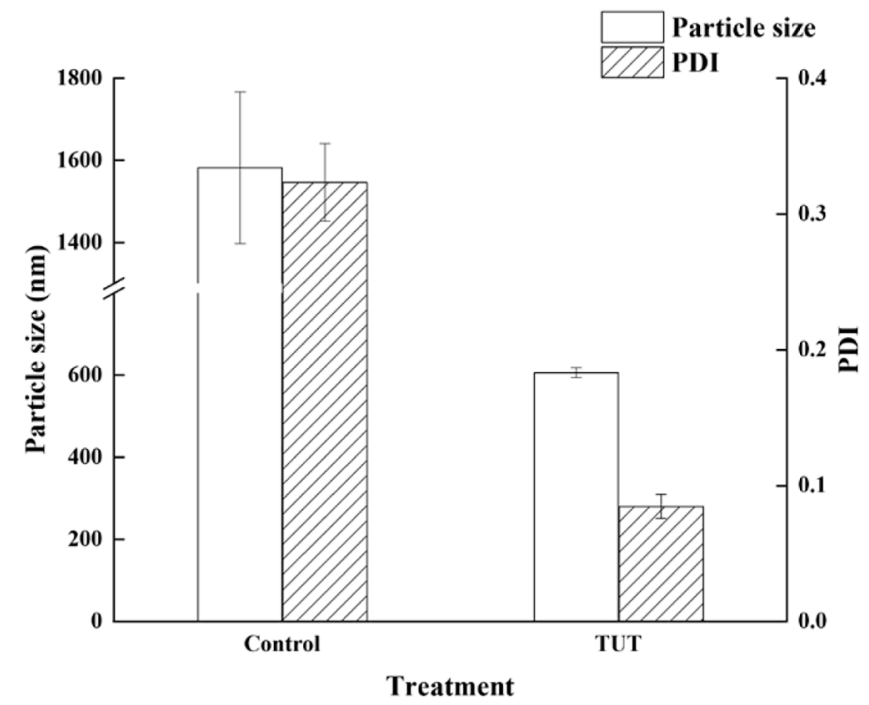

Fig. 3. The particles size and PDI of the halibut bone soup.

It could be clearly seen from the heat map, the TUT treatment could not only significantly increase the content of fatty acids in the bone soup, but also could obtain more types of fatty acids (especially MUFA and PUFA). The fatty acids content in TUT group $(1779.7 \mathrm{mg} / 100 \mathrm{~mL})$ was 14 times higher than that in control group $(126.8 \mathrm{mg} / 100 \mathrm{~mL})$. The shock wave and microjet generated by ultrasound cause damage to biological tissues [33], causing more fatty acids to migrate into the soup. In addition, the perforation caused by ultrasound has mechanical, chemical and shear stresses on the cell membrane. The stress encouraged the formation of holes in the cell membrane, and fatty acid molecules were passively diffused into the soup [34].

\subsection{Effect of TUT on color of the halibut bone soup}

Color is one of the important sensory indicators for judging the quality of bone broth. It can be seen from Table 1 that the $L$ value (80.90) of the bone soup in the TUT group was significantly higher than that of the control group (38.47). At the same time, its $b$ * value was slightly increased ( -2.77 to 0.85 ), and $a^{*}$ value was slightly decreased $(-0.37$ to -0.94). Compared with L value, $a *$ and $b$ * had little change range, so they had little influence on the color of bone broth. From these results, we can infer that TUT can greatly change the color characteristics of bone soup, making it a higher whiteness and brightness. This phenomenon may be related to the improvement of the homogeneity of bone soup by TUT [6]. The cavitation effect of TUT caused the clumps of fat in the bone soup to break into smaller fat globules, making the overall system of bone soup more uniform. In addition, TUT also promoted lipid migration in bone broth, which altered the light dispersion of bone broth and made it noticeably white [33].

\subsection{Formation, properties, and microstructure of MNPs in the halibut bone soup}

\subsubsection{Effect of TUT on particle size of MNPs in halibut bone soup}

The size of MNPs in the emulsion can directly affect the surface properties of MNPs, which in turn determines their functional, physical and structural properties [35]. The results showed that TUT could greatly reduce the particle size of MNPs in bone soup (Fig. 3). Compared with the control group's particle size of $1582.28 \pm 184.79 \mathrm{~nm}$, the particle size of MNPs treated with TUT was only $605.92 \pm 12.09 \mathrm{~nm}$. PDI
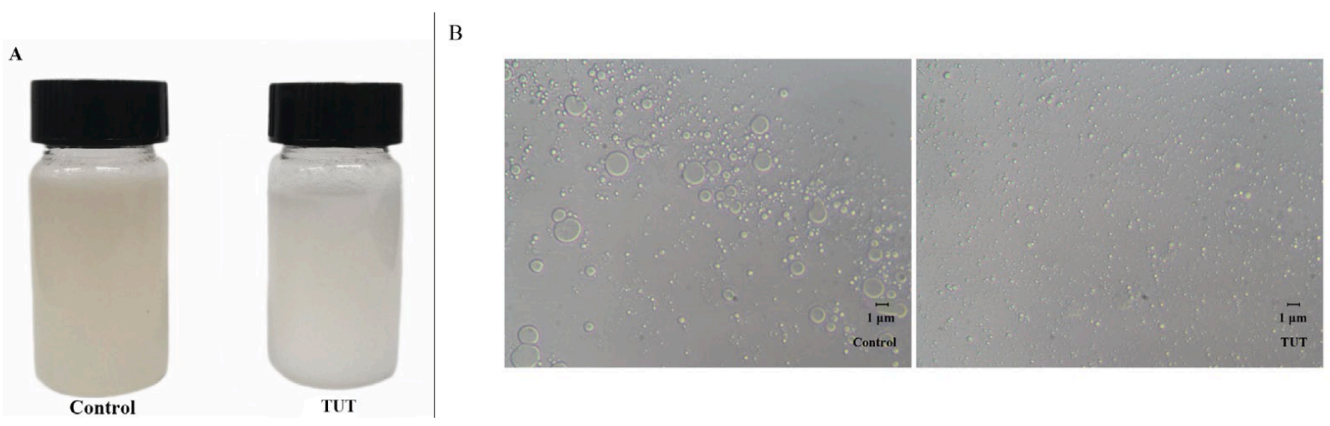

Fig. 4. Appearance and microscopic observation of MNPs in the halibut bone soup. 


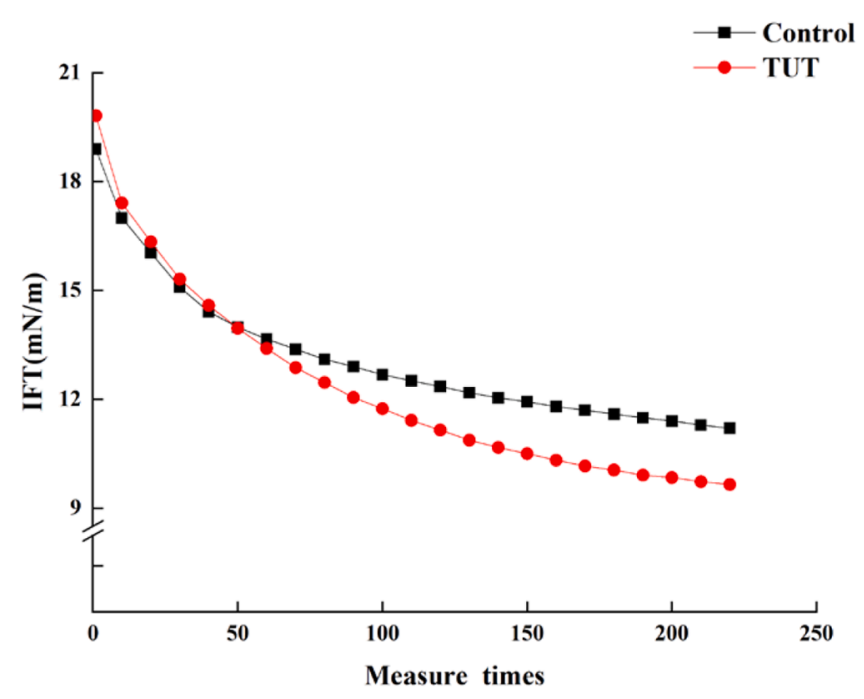

Fig. 5. Changes of the interfacial tension of the halibut bone soup droplets.

was also reduced from 0.32 to 0.08 . These indicated that the particle size distribution of MNPs in the control group was wide, and the whole system was complex and in a chaotic state. The size distribution of MNPs in the TUT group was more concentrated and uniform. This may be because the combined response of TUT cavitation and acoustic flow increased the collision rate and collision strength of MNPs in the bone broth system. The intermolecular hydrophobic interaction between the particles was destroyed [36], which reduced the size of the aggregates of nutrients like proteins and lipids. The PDI was correspondingly narrowed. It could also be seen from the changes in particle size and PDI that TUT contributed to the homogenization of the particle size of MNPs in bone broth and the stability of the entire system.

\subsubsection{Appearance and microscopic observation}

In order to study the influence of TUT on the morphology of halibut bone soup, the appearance and microscopic morphology of MNPs in bone soup emulsion were observed with a camera and an optical microscope. As shown in Fig. 4A, from a macro point of view, the bone broth of the control group was dull and yellow in color, with slight stratification. The bone broth of the TUT group was thick and milky white with uniform texture. The optical micrograph (Fig. 4B) showed the difference in morphology between the two groups on a microscopic level. The MNPs in the two groups of samples were all spherical. Compared with the TUT sample, the control sample had a larger diameter and different sizes. The MNPs in the TUT samples were uniform in size and densely arranged as a whole. This was also consistent with the identification results of color difference and particle size. Therefore, TUT can effectively improve the homogeneity of MNPs in bone broth and promote the stability of the system.

\subsubsection{Effect of TUT on interfacial tension of the halibut bone soup}

As one of the methods to characterize the interfacial behavior of emulsion, interfacial tension is of great significance to study the aggregation behavior and stability of particles in bone broth at oil-water interface [37]. The dynamic changes of interfacial tension at the oil/ water interface of halibut bone soup under different treatments were studied (Fig. 5). It can be seen that the interfacial tension of the two droplets showed a decreasing trend at the initial stage of measurement, then gradually tended to be gentle until stable. This phenomenon may be caused by the diffusion of small molecules from the droplet to the oil-water interface, and then the structural rearrangement of proteins adsorbed on the interface [38]. When the diffusion of molecules at the interfacial layer became saturated, the interaction between the interfacial layers became stable. Meanwhile, the interfacial tension of droplets

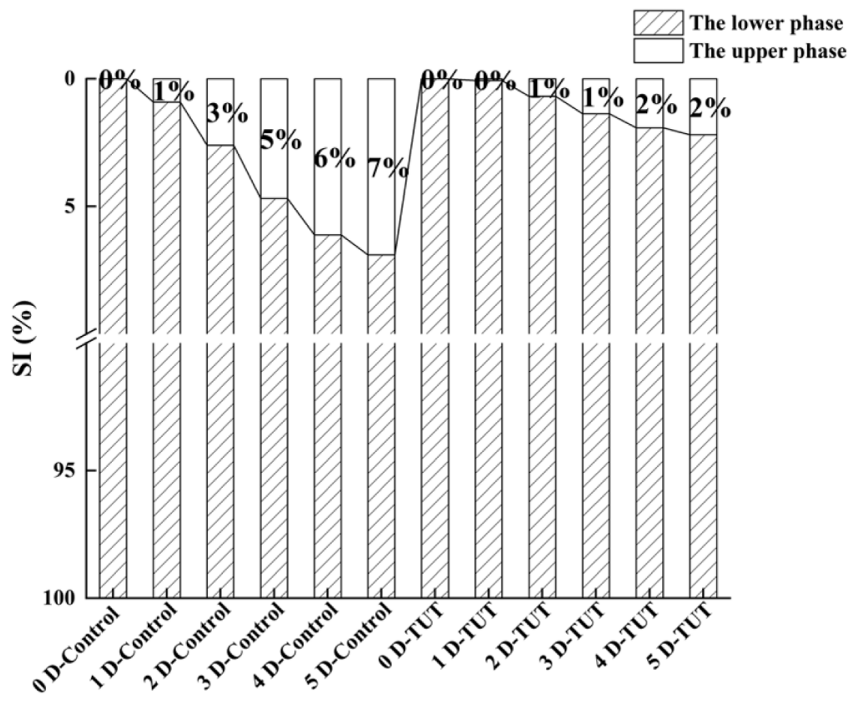

Fig. 6. Dynamic stability of the halibut bone soup.

in the TUT group decreased faster, and the final stable interfacial tension was lower than that in the control group. This indicates that TUT promotes the fragmentation of lipid-protein aggregates in the broth. The cavitation bubbles between the oil and water phase collapse along the contact surface [39], resulting in a lower energy and higher stability of the broth emulsion.

\subsubsection{Effect of TUT on kinetic stability of the halibut bone soup}

The influence of TUT on the kinetic stability of halibut bone soup was shown in Fig. 6. The SI values of both groups increased with the extension of storage time, indicating that the kinetic stability decreased. Among them, the SI of the control group increased significantly on the first day. During the storage period of 5 days, the halibut bone soup had obvious stratification. Compared with the control group, the increase of SI in the TUT group was lower, only $2 \%$ during the 5-day storage period. The results showed that the effect of TUT homogenous enhancement significantly improved the dynamic stability of halibut bone soup. The main reason was that TUT reduced the size of MNPs particles in halibut bone soup. The contact area between MNPs and the intermolecular force, so as to improve the emulsification effect of bone broth system $[40,41]$.

\subsection{Effect of TUT on volatile flavor substances of the halibut bone soup}

\subsubsection{E-nose determination of halibut bone soup}

In order to study the difference in odor profile of halibut bone soup under different treatments, e-nose was used to describe it. The radar fingerprint of the e-nose response value of the bone soup under different treatments was shown in Fig. 7A. Each sensor of the e-nose has obvious response to each sample, and the TUT has a significant effect on the overall odor profile of the bone broth. In general, sensors W5S (nitrogen oxide sensitive), W2S (ethanol sensitive), W3S (alkane sensitive) and W1S (methyl compound sensitive) have higher response values. Thus, halibut bone soup was rich in volatile compounds such as ketones, aldehydes and alcohols. This is consistent with the results that volatile flavor substances such as aldehydes, ketones and alcohols are usually contained in fish products [42]. However, the response value of W5S in the TUT group was significantly increased, while the response value of W2S, W3S and W1S decreased. It is speculated that TUT may promote the conversion of volatile flavor substances and increase the content of nitrogen and oxygen compounds in the bone soup. In order to further analyze the flavor difference of the bone soup at different cooking time points, PCA analysis was performed. As shown in Fig. 7B. PC1 is 93.74\%, 

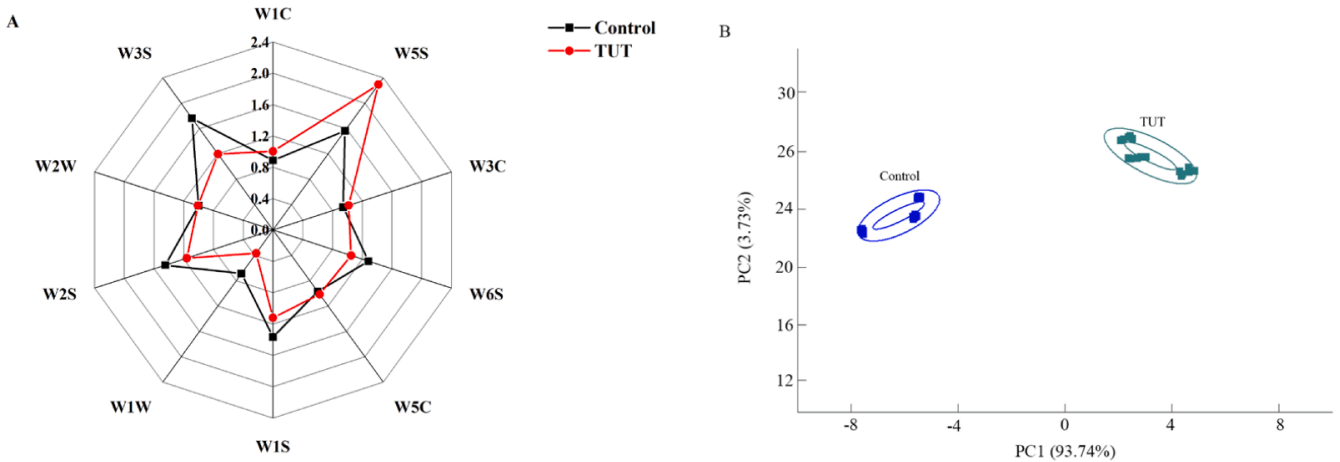

Fig. 7. Radar chart (A) and PCA (B) by e-nose of the halibut bone soup.

Table 2

Identification results of volatile substances in the halibut bone soup.

\begin{tabular}{|c|c|c|c|c|c|c|c|c|}
\hline Count & Compound & CAS\# & Formula & MW & RI & Rt [sec] & Dt [RIPrel] & Comment \\
\hline 1 & Ethanol & C64175 & $\mathrm{C}_{2} \mathrm{H}_{6} \mathrm{O}$ & 46.1 & 515.0 & 95.175 & 1.04726 & \\
\hline 2 & 2-Butanone & C78933 & $\mathrm{C}_{4} \mathrm{H}_{8} \mathrm{O}$ & 72.1 & 582.3 & 125.03 & 1.07244 & \\
\hline 3 & Butanal & C123728 & $\mathrm{C}_{4} \mathrm{H}_{8} \mathrm{O}$ & 72.1 & 597.0 & 131.549 & 1.2925 & \\
\hline 4 & Ethyl Acetate & C141786 & $\mathrm{C}_{4} \mathrm{H}_{8} \mathrm{O}_{2}$ & 88.1 & 602.0 & 133.811 & 1.3368 & Dimer \\
\hline 5 & Ethyl Acetate & C141786 & $\mathrm{C}_{4} \mathrm{H}_{8} \mathrm{O}_{2}$ & 88.1 & 607.3 & 136.152 & 1.09901 & Monomer \\
\hline 6 & Acetic acid & C64197 & $\mathrm{C}_{2} \mathrm{H}_{4} \mathrm{O}_{2}$ & 60.1 & 628.4 & 145.518 & 1.06544 & \\
\hline 7 & 1-Hydroxy-2-propanone & C116096 & $\mathrm{C}_{3} \mathrm{H}_{6} \mathrm{O}_{2}$ & 74.1 & 646.9 & 153.714 & 1.03607 & \\
\hline 8 & 3-Methylbutanal & C590863 & $\mathrm{C}_{5} \mathrm{H}_{10} \mathrm{O}$ & 86.1 & 652.5 & 156.203 & 1.4033 & \\
\hline 9 & 2-Methylbutanal & C96173 & $\mathrm{C}_{5} \mathrm{H}_{10} \mathrm{O}$ & 86.1 & 654.8 & 157.226 & 1.16755 & \\
\hline 10 & 2-Pentanone & C107879 & $\mathrm{C}_{5} \mathrm{H}_{10} \mathrm{O}$ & 86.1 & 679.3 & 168.087 & 1.12236 & \\
\hline 11 & 3-Pentanone & C96220 & $\mathrm{C}_{5} \mathrm{H}_{10} \mathrm{O}$ & 86.1 & 691.4 & 174.454 & 1.37979 & \\
\hline 12 & Pentanal & C110623 & $\mathrm{C}_{5} \mathrm{H}_{10} \mathrm{O}$ & 86.1 & 694.1 & 176.544 & 1.18993 & Monomer \\
\hline 13 & Pentanal & C110623 & $\mathrm{C}_{5} \mathrm{H}_{10} \mathrm{O}$ & 86.1 & 694.9 & 177.129 & 1.42631 & Dimer \\
\hline 14 & Propyl acetate & C109604 & $\mathrm{C}_{5} \mathrm{H}_{10} \mathrm{O}_{2}$ & 102.1 & 705.4 & 185.325 & 1.47946 & Dimer \\
\hline 15 & Propyl acetate & C109604 & $\mathrm{C}_{5} \mathrm{H}_{10} \mathrm{O}_{2}$ & 102.1 & 707.6 & 187.081 & 1.16755 & Monomer \\
\hline 16 & Methyl isobutyl ketone & C108101 & $\mathrm{C}_{6} \mathrm{H}_{12} \mathrm{O}$ & 100.2 & 729.3 & 204.057 & 1.18154 & \\
\hline 17 & 2-Pentenal & C764396 & $\mathrm{C}_{5} \mathrm{H}_{8} \mathrm{O}$ & 84.1 & 736.8 & 209.911 & 1.09342 & Monomer \\
\hline 18 & 2-Pentenal & C764396 & $\mathrm{C}_{5} \mathrm{H}_{8} \mathrm{O}$ & 84.1 & 737.6 & 210.497 & 1.35358 & Dimer \\
\hline 19 & (E)-2-Pentenal & C1576870 & $\mathrm{C}_{5} \mathrm{H}_{8} \mathrm{O}$ & 84.1 & 751.1 & 221.034 & 1.36337 & Dimer \\
\hline 20 & (E)-2-Pentenal & C1576870 & $\mathrm{C}_{5} \mathrm{H}_{8} \mathrm{O}$ & 84.1 & 751.8 & 221.619 & 1.10741 & Monomer \\
\hline 21 & 1-Pentanol & C71410 & $\mathrm{C}_{5} \mathrm{H}_{12} \mathrm{O}$ & 88.1 & 772.0 & 237.424 & 1.25287 & \\
\hline 22 & 2-Hexanone & C591786 & $\mathrm{C}_{6} \mathrm{H}_{12} \mathrm{O}$ & 100.2 & 775.6 & 240.242 & 1.18113 & \\
\hline 23 & Hexanal & C66251 & $\mathrm{C}_{6} \mathrm{H}_{12} \mathrm{O}$ & 100.2 & 792.3 & 255.914 & 1.25613 & Monomer \\
\hline 24 & Hexanal & C66251 & $\mathrm{C}_{6} \mathrm{H}_{12} \mathrm{O}$ & 100.2 & 792.9 & 256.62 & 1.56013 & Dimer \\
\hline 25 & Butyl acetate & C123864 & $\mathrm{C}_{6} \mathrm{H}_{12} \mathrm{O}_{2}$ & 116.2 & 805.0 & 270.033 & 1.23333 & \\
\hline 26 & Butanoic acid & C107926 & $\mathrm{C}_{4} \mathrm{H}_{8} \mathrm{O}_{2}$ & 88.1 & 823.4 & 290.505 & 1.15733 & \\
\hline 27 & 2-Methylbutanoic acid & C116530 & $\mathrm{C}_{5} \mathrm{H}_{10} \mathrm{O}_{2}$ & 102.1 & 828.5 & 296.152 & 1.20445 & \\
\hline 28 & (E)-2-Hexenal & C6728263 & $\mathrm{C}_{6} \mathrm{H}_{10} \mathrm{O}$ & 98.1 & 846.3 & 315.918 & 1.18165 & Monomer \\
\hline 29 & (E)-2-Hexenal & $\mathrm{C} 6728263$ & $\mathrm{C}_{6} \mathrm{H}_{10} \mathrm{O}$ & 98.1 & 846.9 & 316.624 & 1.51605 & Dimer \\
\hline 30 & 1-Hexanol & C111273 & $\mathrm{C}_{6} \mathrm{H}_{14} \mathrm{O}$ & 102.2 & 875.7 & 348.648 & 1.32421 & \\
\hline 31 & 2-Heptanone & C110430 & $\mathrm{C}_{7} \mathrm{H}_{14} \mathrm{O}$ & 114.2 & 889.9 & 364.454 & 1.26266 & \\
\hline 32 & Cyclohexanone & C108941 & $\mathrm{C}_{6} \mathrm{H}_{10} \mathrm{O}$ & 98.1 & 898.2 & 377.334 & 1.14821 & \\
\hline 33 & Heptanal & C111717 & $\mathrm{C}_{7} \mathrm{H}_{14} \mathrm{O}$ & 114.2 & 900.3 & 380.864 & 1.33973 & Monomer \\
\hline 34 & Heptanal & C111717 & $\mathrm{C}_{7} \mathrm{H}_{14} \mathrm{O}$ & 114.2 & 900.3 & 380.864 & 1.69389 & Dimer \\
\hline 35 & alpha-Pinene & C80568 & $\mathrm{C}_{10} \mathrm{H}_{16}$ & 136.2 & 928.3 & 428.867 & 1.21509 & \\
\hline 36 & Methyl hexanoate & C106707 & $\mathrm{C}_{7} \mathrm{H}_{14} \mathrm{O}_{2}$ & 130.2 & 934.5 & 439.456 & 1.30781 & \\
\hline 37 & (E)-2-Heptenal & C18829555 & $\mathrm{C}_{7} \mathrm{H}_{12} \mathrm{O}$ & 112.2 & 955.5 & 475.458 & 1.25765 & \\
\hline 38 & Benzaldehyde & C100527 & $\mathrm{C}_{7} \mathrm{H}_{6} \mathrm{O}$ & 106.1 & 957.5 & 478.988 & 1.15277 & \\
\hline 39 & 1-Octen-3-ol & C3391864 & $\mathrm{C}_{8} \mathrm{H}_{16} \mathrm{O}$ & 128.2 & 976.3 & 511.072 & 1.14839 & \\
\hline 40 & Octanal & C124130 & $\mathrm{C}_{8} \mathrm{H}_{16} \mathrm{O}$ & 128.2 & 1010.4 & 574.436 & 1.40522 & Monomer \\
\hline 41 & Octanal & C124130 & $\mathrm{C}_{8} \mathrm{H}_{16} \mathrm{O}$ & 128.2 & 1011.1 & 575.756 & 1.82145 & Dimer \\
\hline 42 & (E)-2-Octenal & C2548870 & $\mathrm{C}_{8} \mathrm{H}_{14} \mathrm{O}$ & 126.2 & 1063.6 & 683.983 & 1.33325 & \\
\hline 43 & Nonanal & C124196 & $\mathrm{C}_{9} \mathrm{H}_{18} \mathrm{O}$ & 142.2 & 1103.3 & 765.813 & 1.47913 & \\
\hline 44 & 2-Decanone & C693549 & $\mathrm{C}_{10} \mathrm{H}_{20} \mathrm{O}$ & 156.3 & 1151.4 & 864.854 & 1.46317 & \\
\hline
\end{tabular}

PC2 is $3.73 \%$, and the total contribution rate is $97.17 \%$, which is greater than $95 \%$. It is credible and can represent the main characteristics of volatile flavor in the sample [22]. In terms of spatial distribution, the odor contours of the control group and the TUT group are completely separated, and there is no overlap. It indicated that the two samples have a certain degree of distinction and can be clearly identified.

\subsubsection{HS-GC-IMS determination}

As shown in Table 2 , a total of 51 volatile substances were detected from the two groups of halibut bone soup samples, 44 of which were qualitatively identified by HS-GC-IMS database. It included 21 aldehydes, 4 alcohols, 6 esters, 9 ketones, 3 acids, and 1 other type of compound. Some compounds even produced monomer and dimer signals. It might be due to the formation of adducts between ions and neutral molecules, which were separated as the material passes through 


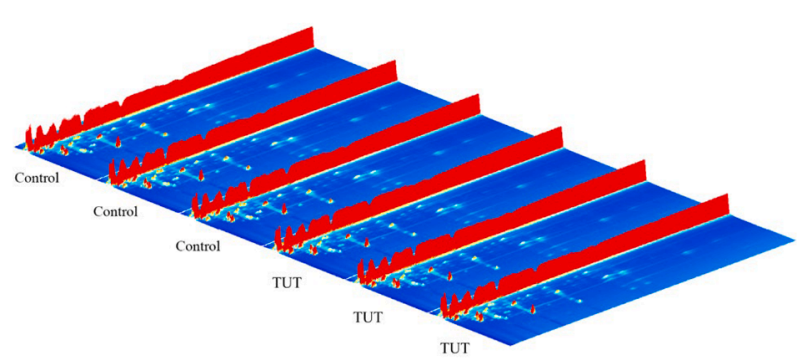

Fig. 8. 3D spectrum of HS-GC-IMS.

the drift region [43]. Fig. 8 showed the 3D spectra of volatile substances in the two samples. This graph shows the difference of volatile flavor compounds between two groups of halibut bone soup. Fig. 9 was obtained by fingerprint analysis of different substances according to the spectrum. The rows and columns represent all the signal peaks of a sample and the same volatile substance in different samples.

It can be seen from the fingerprint that the main volatile flavor compounds in halibut bone soup were aldehydes and ketones, of which aldehydes dominate. The control group had relatively high aldehyde content, mainly valeraldehyde, hexanal, heptanal, and nonanal. Among them, hexanal is related to fishy, grassy, rancid and other unpleasant odors. Beyond that, 1-octen-3-ol produces fishy smell and odor. In addition, the acid content in the control group was relatively higher. For example, acetic acid, butyric acid, and 2-methylbutyric acid have a pungent and unpleasant odor. However, the contents of most volatile flavor substances in halibut bone soup were increased in TUT, but the contents of aldehyde and acid substances in halibut bone soup with fishy, earthy and irritant odor were significantly decreased. TUT promoted the esterification of alcohol and carboxylic acid in halibut bone soup [44], resulting in the increase of ethyl acetate, propyl acetate, butyl caproate and other esters. These substances usually have a fruity aroma and help improve the overall flavor of halibut bone soup. In addition, the formation of MNPs may also contribute to the change of volatile flavor profile. Since the emulsification system of halibut bone soup contained proteins, lipids and polysaccharides, MNPs have a certain ability to adsorb volatile flavor substances. The MNPS particles in the bone soup of the control group were large and different sizes, and the overall system was relatively chaotic. The oil with volatile flavor and greater binding ability floated in uneven distribution [45], resulting in a relatively unsatisfactory flavor profile. However, TUT made MNPs more uniform in size and made the nutrients uniformly distributed in the whole bone broth system. Meanwhile, the decrease of particle size led to the increase of the adsorption interface area and the adsorption capacity of MNPs for volatile flavor substances [46].

\subsection{Effect of TUT on taste substances of the halibut bone soup}

\subsubsection{Effect of TUT on $5^{\prime}$ - nucleotides and organic acids of the halibut bone soup}

The distinctive taste produced by glutamate is called umami. The $5^{\prime}$ nucleotide can also play an important role in enhancing umami flavor [47]. As shown in Table 3, two 5'-nucleotides, namely 5'-GMP and 5'AMP, were detected in both sets of samples. Taste activity value (TAV) is calculated by the ratio of the taste activity value measured in the sample to the threshold value measured in water or in a simple matrix. TAV is often used to evaluate the taste contribution of compounds, and a TAV greater than 1 indicates that TAV has a significant effect on food taste [49]. The results showed that the nucleotide content of $5^{\prime}$-GMP (Control vs TUT $=24.70 \pm 0.21 \mathrm{mg} / 100 \mathrm{~mL}$ vs $39.95 \pm 0.32 \mathrm{mg} / 100 \mathrm{~mL})$ was higher than that of $5^{\prime}$-AMP (Control vs TUT $=4.39 \pm 0.19 \mathrm{mg} / 100 \mathrm{~mL}$ vs $4.97 \pm 0.25 \mathrm{mg} / 100 \mathrm{~mL}$ ). At the same time, TAV values of $5^{\prime}$-GMP were all greater than 1 , indicating that the existence of $5^{\prime}$-GMP had an important effect on the taste of halibut bone soup. The TAV value of $5^{\prime}$ AMP was less than 1 , and its effect on taste was negligible compared with $5^{\prime}$-GMP. In addition, it can be seen from the table that the content of $5^{\prime}$-nucleotide in the control group was significantly lower than that in the TUT group. These indicated that TUT can also promote the dissolution of $5^{\prime}$-nucleotide, especially the dissolution of $5^{\prime}$-GMP, which has a positive effect on the umami of bone soup.

As can be seen from Table 3, lactic acid, citric acid and succinic acid are three organic acids in all samples. These organic acids not only have sour taste, but also provide umami taste. Succinic acid, as one of the main taste active ingredients of most seafood, provides unique sour and umami taste to seafood [50]. Lactic acid is an important flavor substance in fermented food and the main organic acids in aquatic products [51]. Citric acid provides a mild, refreshing acidity to the taste of seafood. In the control group, the content of lactic acid was the highest $(12.38 \mathrm{mg} /$ $\mathrm{mL})$, and citric acid was the lowest $(0.67 \mathrm{mg} / \mathrm{mL})$. However, due to the low threshold value of succinic acid, which is only 0.11 , the TAV value of succinic acid is the highest, reaching 25.39, much higher than that of

Table 3

Changes in $5^{\prime}$-nucleotide and organic acids contents of the halibut bone soup.

\begin{tabular}{lcll}
\hline Item & Threshold value* & Content & \\
\cline { 3 - 4 } & & Control (TAV) & TUT (TAV) \\
\hline 5'-Nucleotides (mg/100 mL) & & \\
5'-GMP & 12.5 & $24.70 \pm 0.21(1.88)$ & $39.95 \pm 0.32(2.18)$ \\
5'-AMP & 50 & $4.39 \pm 0.19(0.09)$ & $4.97 \pm 0.25(0.1)$ \\
Total & & & 44.92 \\
Organic acids (mg/mL) & & \\
Succinic acid $\quad 0.11$ & $2.79 \pm 0.21(25.39)$ & $9.00 \pm 0.17(81.82)$ \\
Lactic acid & 1.26 & $12.38 \pm 0.70(9.87)$ & $7.33 \pm 0.53(5.82)$ \\
Citric acid & 0.45 & $0.67 \pm 0.06(1.49)$ & $3.56 \pm 0.07(7.91)$ \\
Total & & 16.78 & 19.89 \\
\hline
\end{tabular}

Thresholds were mainly gathered from the following literature $[47,48]$.

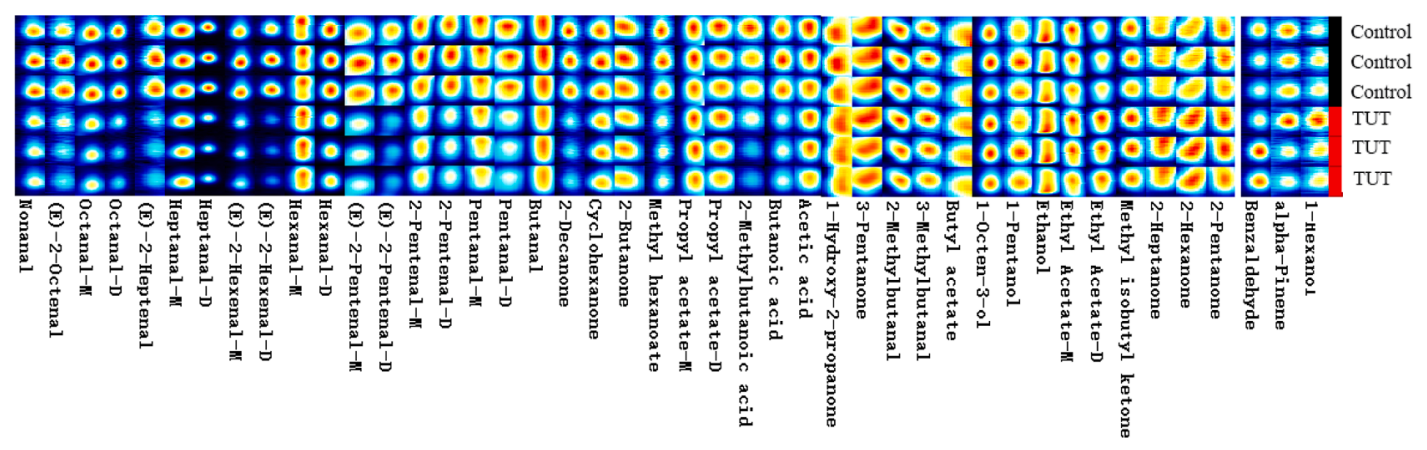

Fig. 9. Fingerprint determination of the halibut bone soup by HS-GC-IMS. 


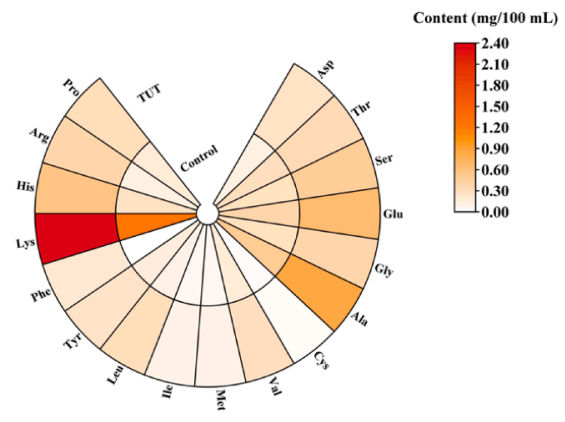

B

\begin{tabular}{|c|c|c|c|}
\hline o & O & Umami & Content $(\mathrm{mg} / 100 \mathrm{~mL})$ \\
\hline 0 & 0 & Sweet & O 2.00 \\
\hline 0 & 0 & Bitter & 6.00 \\
\hline$\circ$ & $\circ$ & Salt & 8.00 \\
\hline$\circ$ & 0 & Sour & \\
\hline & & TAA & \\
\hline
\end{tabular}

Fig. 10. Heat map of free amino acids content of halibut bone soup (A) and the content of different type of FAAs (B).

lactic acid and citric acid (9.87 \& 1.49). So succinic acid plays a key role in the umami taste of halibut bone broth, while lactic acid and citric acid also contribute to the taste of the broth. In addition, the contents of succinic acid $(9 \mathrm{mg} / \mathrm{mL})$ and citric acid $(3.56 \mathrm{mg} / \mathrm{mL})$ were increased by TUT, but the contents of lactic acid $(7.33 \mathrm{mg} / \mathrm{mL})$ were decreased. Succinic acid still made the largest contribution to the taste of the sample, while the TAV of citric acid increased, making its contribution to the taste more than lactic acid, although the content of citric acid was still lowest.

In general, TUT can increase the content of $5^{\prime}$-nucleotides and organic acids and enrich the flavor substances in the samples.

\subsubsection{Effect of TUT on FAA of the halibut bone soup}

FAAs, as indispensable taste and precursor substances in aquatic products, and also play a vital role in the formation of the halibut bone soup taste. Fig. $10 \mathrm{~A}$ is the heat map of FAAs composition and content (Table S2). As can be seen, FAAs levels in the TUT group were significantly higher than those in the control group. The contents of amino acids in all tastes were increased, and the increase of bitter amino acids was more obvious (Fig. 10 B). The cavitation and crushing effect of TUT on raw materials caused FAAs in tissues to migrate to the soup. In addition, the denaturation of protein was more serious under the combined action of ultrasound and heat treatment, and the FAAs content increased significantly. However, as the TAV values of FAAs were very low in general, far less than 1 , the increase of FAAs, especially the increase of bitter amino acids, had little effect on the taste profile of the halibut bone soup.

\section{Conclusion}

In this paper, the conventional heating method and the TUT method were used to cook the halibut bone soup. TUT had obvious optimization effect on the quality of the fish bone soup. Compared with the control group, the contents of total sugars, water-soluble proteins, fatty acids and solids in the TUT group were higher, and the MNPs were smaller and more uniform, thus the interfacial tension of the bone soup was lower, and the emulsification effect and stability were better. In terms of flavor profile, the content of flavor substances in the TUT group was more abundant, and the volatile flavor was more harmonious. These optimizations are achieved through the destruction of raw material tissues and cells by the shear, cavitation and homogenization effects of TUT.

\section{CRediT authorship contribution statement}

Wenhui Zhu: Writing - original draft. Wei He: Writing - original draft. Wenxuan Wang: Project administration. Ying Bu: Writing - review \& editing. Xuepeng Li: Data curation. Jianrong Li: Methodology. Yuyu Zhang: Validation.

\section{Declaration of Competing Interest}

The authors declare that they have no known competing financial interests or personal relationships that could have appeared to influence the work reported in this paper.

\section{Acknowledgements}

This study was supported by the Scientific Research Project of Liaoning Education Department (LJKZ1017), National Natural Science Foundation of China (31901763), and Open Project Program of Beijing Key Laboratory of Flavor Chemistry (SPFW2021YB01).

\section{Appendix A. Supplementary data}

Supplementary data to this article can be found online at https://doi. org/10.1016/j.ultsonch.2021.105785.

\section{References}

[1] L. Zhou, K. Nyberg, A.C. Rowat, Understanding diffusion theory and fick's law through food and cooking, Adv. Physiol. Educ. 39 (3) (2015) 192-197, https://doi. org/10.1152/advan.00133.2014.

[2] L. Ke, J. Zhou, L. Wei, G. Gao, P. Rao, The power of soups: super-hero or teamwork? Trends Food Sci. Technol. 22 (9) (2011) 492-497, https://doi.org/10.1016/ j.tifs.2011.06.004.

[3] L. Ke, H. Wang, G. Gao, P. Rao, L. He, J. Zhou, Direct interaction of food derived colloidal micro/nano-particles with oral macrophages, NPJ Sci. Food 1 (1) (2017) 3, https://doi.org/10.1038/s41538-017-0003-3.

[4] M. Orrego, E. Troncoso, R.N. Zuniga, Aerated whey protein gels as new food matrices: effect of thermal treatment over microstructure and textural properties, J. Food Eng. 163 (2015) 37-44, https://doi.org/10.1016/j.jfoodeng.2015.04.027.

[5] J. Zhou, L. Jie, L. Dai, G. Gao, H. Wang, J. Guo, P. Rao, L. Ke, Boiling-induced nanoparticles and their constitutive proteins from isatis indigotica fort. root decoction: purification and identification, Journal of Traditional and Complementary Medicine. 7(2) (2017) 178-187. https://doi.org/10.1016/j. jtcme.2016.08.007.

[6] L.M. Carrillo-Lopez, I.A. Garcia-Galicia, J.M. Tirado-Gallegos, R. Sanchez-Vega, M. Huerta-Jimenez, M. Ashokkumar, A.D. Alarcon-Rojo, Recent advances in the application of ultrasound in dairy products: effect on functional, physical, chemical, microbiological and sensory properties, Ultrason. Sonochem. 73 (2021) 105467, https://doi.org/10.1016/j.ultsonch.2021.105467.

[7] S. Wang, J. Kang, X. Zhang, Z. Guo, Dendrites fragmentation induced by oscillating cavitation bubbles in ultrasound field, Ultrasonics 83 (2018) 26-32, https://doi. org/10.1016/j.ultras.2017.08.004.

[8] M. Ashokkumar, The characterization of acoustic cavitation bubbles - an overview, Ultrason. Sonochem. 18 (4) (2011) 864-872, https://doi.org/10.1016/j. ultsonch.2010.11.016.

[9] B.K. Tiwari, Ultrasound: a clean, green extraction technology, TrAC, Trends Anal. Chem. 71 (2015) 100-109, https://doi.org/10.1016/j.trac.2015.04.013.

[10] M.G.M. Costa, T.V. Fonteles, A.L.T. de Jesus, F.D.L. Almeida, M.R.A. de Miranda, F. A.N. Fernandes, S. Rodrigues, High-intensity ultrasound processing of pineapple juice, Food Bioprocess Technol. 6 (4) (2013) 997-1006, https://doi.org/10.1007/ s11947-011-0746-9.

[11] M. Singla, N. Sit, Application of ultrasound in combination with other technologies in food processing: a review, Ultrason. Sonochem. 73 (6) (2021) 105506-105519, https://doi.org/10.1016/j.ultsonch.2021.105506.

[12] J.A. Téllez-Morales, B. Hernández-Santo, J. Rodríguez-Miranda, Effect of ultrasound on the techno-functional properties of food components/ingredients: a 
review, Ultrason. Sonochem. 61 (2020) 104787, https://doi.org/10.1016/j. ultsonch.2019.104787.

[13] Y. Sui, W.-C. Huang, Y. Wu, X. Qi, X. Mao, Lipid extraction from Greenland halibut (reinhardtius hippoglossoides) by-product in low-voltage dc electric field and its mechanism, J. Cleaner Prod. 283 (2021) 124673, https://doi.org/10.1016/j. jclepro.2020.124673.

[14] D. Sohn, L. Ciannelli, J.T. Duffy-Anderson, Distribution of early life pacific halibut and comparison with Greenland halibut in the eastern bering sea, J. Sea Res. 107 (2016) 31-42, https://doi.org/10.1016/j.seares.2015.09.001.

[15] H. Karl, M. Lahrssen-Wiederholt, Factors influencing the intake of dioxins and dioxin-like PCBs via fish consumption in Germany, J. Verbraucherschutz Lebensmittelsicherheit. 8 (1-2) (2013) 27-35, https://doi.org/10.1007/s00003013-0805-4.

[16] F. Leng, S. Sun, Y. Jing, F. Wang, Q. Wei, X. Wang, X. Zhu, A rapid and sensitive method for determination of trace amounts of glucose by anthrone-sulfuric acid method, Bul. Chem. Commun. 48 (1) (2016) 109-113.

[17] E. Subroto, E. Lembong, F. Filianty, R. Indiarto, S. Junar, The analysis techniques of amino acid and protein in food and agricultural products, Int. J. Sci. Technol. Res. 9 (10) (2020) 29-36.

[18] M. M. Zcan, M. S. Alkaltham, N. Uslu, A. Salamatullah, Effect of different roasting methods on the bioactive properties, phenolic compounds and fatty acids compositions of pomegranate (Punica granatum L. cv. Hicaz) seed and oils, Journal of Food Science and Technology. 58(6) (2020) 2283-2294. https://doi.org/ 10.1007/s13197-020-04739-1.

[19] X.-W. Chen, S.-D. Sun, C.-G. Ma, X.-Q. Yang, Oil-water interfacial-directed spontaneous self-assembly of natural quillaja saponin for controlling interface permeability in colloidal emulsions, J. Agric. Food. Chem. 68 (47) (2020) 13854-13862, https://doi.org/10.1021/acs.jafc.0c04431.

[20] S. Fang, T. Chen, R. Wang, Y. Xiong, B. Chen, M. Duan, Assembly of graphene oxide at the crude oil/water interface: a new approach to efficient demulsification, Energy Fuels 30 (4) (2016) 3355-3364, https://doi.org/10.1021/acs. energyfuels.6b00195.

[21] M. Wang, C. Fan, K. Zhao, B. Huang, H. Hu, X. Xu, S. Pan, F. Liu, Characterization and functional properties of mango peel pectin extracted by ultrasound assisted citric acid, Int. J. Biol. Macromol. 91 (2016) 794-803, https://doi.org/10.1016/j. ijbiomac.2016.06.011.

[22] W. Zhu, H. Luan, Y. Bu, X. Li, J. Li, G. Ji, Flavor characteristics of shrimp sauces with different fermentation and storage time, LWT- Food Sci. Technol. 110 (2019) 142-151, https://doi.org/10.1016/j.lwt.2019.04.091.

[23] K. Chen, X. Yang, Z. Huang, S. Jia, Y. Zhang, J. Shi, H. Hong, L. Feng, Y. Luo, Modification of gelatin hydrolysates from grass carp (Ctenopharyngodon idellus) scales by Maillard reaction: antioxidant activity and volatile compounds, Food Chem. 295 (2019) 569-578, https://doi.org/10.1016/j.foodchem.2019.05.156.

[24] X. Fan, X. Jiao, J. Liu, M. Jia, C. Blanchard, Z. Zhou, Characterizing the volatile compounds of different sorghum cultivars by both GC-MS and HS-GC-IMS, Food Res. Int. 140 (2021) 109975, https://doi.org/10.1016/j.foodres.2020.109975.

[25] Y. Kong, X. Yang, Q. Ding, Y.Y. Zhang, B.G. Sun, H.T. Chen, Y. Sun, Comparison of non-volatile umami components in chicken soup and chicken enzymatic hydrolysate, Food Res. Int. 102 (2017) 559-566, https://doi.org/10.1016/j. foodres.2017.09.038.

[26] X. Xu, M. You, H. Song, L. Gong, W. Pan, Investigation of umami and kokumi tasteactive components in bovine bone marrow extract produced during enzymatic hydrolysis and Maillard reaction, Int. J. Food Sci. Technol. 53 (11) (2018) 2465-2481, https://doi.org/10.1111/ijfs.13893.

[27] Y. Akagündüz, M. Mosquera, B. Giménez, A. Alemán, P. Montero, M.C. GómezGuillén, Sea bream bones and scales as a source of gelatin and ACE inhibitory peptides, LWT - Food Sci. Technol. 55 (2) (2014) 579-585, https://doi.org/ 10.1016/j.lwt.2013.10.026.

[28] M.A.J.S.V. Boekel, Formation of flavour compounds in the Maillard reaction, Biotechnol. Adv. 24 (2) (2006) 230-233, https://doi.org/10.1016/j. biotechadv.2005.11.004.

[29] A. Santos, T.N.H. Rebouas, J. Souza, R. Bonomo, L.M.D. Silva, Characterization and evaluation of quality of soups dehydrated produced with flour of potatoes during time of storage, Boletim Centro de Pesquisa de Processamento de Alimentos. 28 (1) (2010) 57-68.

[30] S.A. Sena, K. Bulent, S. Nihan, B. Banu, U.A. Abdullah, A. Cesarettin, Optimizing the extraction parameters of epigallocatechin gallate using conventional hot water and ultrasound assisted methods from green tea, Food Bioprod. Process. 111 (2018) 37-44, https://doi.org/10.1016/j.fbp.2018.06.003.
[31] X. Wang, Y.u. Huang, B. Zhou, W. Xu, X. Xiang, Q. Huang, S. Li, Improvement of quality and flavor of salted egg yolks by ultrasonic assisted cooking, Ultrason. Sonochem. 75 (2021) 105579, https://doi.org/10.1016/j.ultsonch.2021.105579.

[32] D. Swanson, R. Block, S.A. Mousa, Omega-3 fatty acids epa and dha: health benefits throughout life, Adv. Nutr. 3 (1) (2012) 1-7, https://doi.org/10.3945/ an.111.000893.

[33] A. Visy, G. Jónás, D. Szakos, Z. Horváth-Mezőfi, K.I. Hidas, A. Barkó, L. Friedrich, Evaluation of ultrasound and microbubbles effect on pork meat during brining process, Ultrason. Sonochem. 75 (2021) 105589, https://doi.org/10.1016/j. ultsonch.2021.105589.

[34] I. Siró, Cs. VéN, C. Balla, G. JóNás, I. Zeke, L. Friedrich, Application of an ultrasonic assisted curing technique for improving the diffusion of sodium chloride in porcine meat, Journal of Food Engineering. 91(2) (2009) 353-362. https://doi. org/10.1016/j.jfoodeng.2008.09.015.

[35] K.A. Boora, K. Amarjeet, K.S. Kumar, M. Nitin, Characterization of heat-stable whey protein: impact of ultrasound on rheological, thermal, structural and morphological properties, Ultrason. Sonochem. 49 (2018) 333-342, https://doi. org/10.1016/j.ultsonch.2018.08.026.

[36] A.R. Jambrak, T.J. Mason, V. Lelas, L. Paniwnyk, Z. Herceg, Effect of ultrasound treatment on particle size and molecular weight of whey proteins, J. Food Eng. 121 (2014) 15-23, https://doi.org/10.1016/j.jfoodeng.2013.08.012.

[37] G.L. Maddikeri, P.R. Gogate, A.B. Pandit, Improved synthesis of sophorolipids from waste cooking oil using fed batch approach in the presence of ultrasound, Chem. Eng. J. 263 (2015) 479-487, https://doi.org/10.1016/j.cej.2014.11.010.

[38] A. Koh, R.J. Linhardt, R. Gross, Effect of sophorolipid n-alkyl ester chain length on its interfacial properties at the almond oil-water interface, Langmuir 32 (22) (2016) 5562-5572, https://doi.org/10.1021/acs.langmuir.6b01008.

[39] J.P. Canselier, H. Delmas, A.M. Wilhelm, B. Abismaïl, Ultrasound emulsification-an overview, J. Dispersion Sci. Technol. 23 (1-3) (2002) 333-349.

[40] E.K. Silva, M. Meireles, Influence of the degree of inulin polymerization on the ultrasound-assisted encapsulation of annatto seed oil, Carbohydr. Polym. 133 (2015) 578-586, https://doi.org/10.1016/j.carbpol.2015.07.025.

[41] N. Nguyen, S.G. Anema, Effect of ultrasonication on the properties of skim milk used in the formation of acid gels, Innovative Food Sci. Emerg. Technol. 11 (4) (2010) 616-622, https://doi.org/10.1016/j.ifset.2010.05.006.

[42] W.Q. Qiu, S.S. Chen, J. Xie, Y.H. Qu, X. Song, Analysis of 10 nucleotides and related compounds in litopenaeus vannamei during chilled storage by HPLC-DAD, LWT - Food Sci. Technol. 67 (2016) 187-193, https://doi.org/10.1016/j. lwt.2015.11.047.

[43] R. Rodríguez-Maecker, E. Vyhmeister, S. Meisen, A.R. Martinez, A. Kuklya, U. Telgheder, Identification of terpenes and essential oils by means of static headspace gas chromatography-ion mobility spectrometry, Anal. Bioanal. Chem. 409 (28) (2017) 6595-6603, https://doi.org/10.1007/s00216-017-0613-2.

[44] P. Boraphech, P. Thiravetyan, Removal of trimethylamine (fishy odor) by c3 and cam plants, Environ. Sci. Pollut. Res. 22 (15) (2015) 11543-11557, https://doi. org/10.1007/s11356-015-4364-3.

[45] N. Innocente, G. Marchesini, M. Biasutti, Effect of high-pressure homogenisation on the retention of selected aroma compounds in model dairy emulsions, Int. J. Food Sci. Technol. 49 (9) (2014) 1992-2000, https://doi.org/10.1111/ijfs.2014.49. issue-910.1111/ijfs.12520.

[46] A. Meynier, V. Rampon, M. Dalgalarrondo, C. Genot, Hexanal and t-2-hexenal form covalent bonds with whey proteins and sodium caseinate in aqueous solution, Int. Dairy J. 14 (8) (2004) 681-690, https://doi.org/10.1016/j.idairyj.2004.01.003.

[47] S. Yamaguchi, T. Yoshikawa, S. Ikeda, T. Ninomiya, Measurement of the relative taste intensity of some L-a-amino acids and 5'-nucleotides, J. Food Sci. 36 (6) (1971) 846-849, https://doi.org/10.1111/j.1365-2621.1971.tb15541.x.

[48] J.C. Hufnagel, T. Hofmann, Quantitative reconstruction of the nonvolatile sensometabolome of a red wine, J. Agric. Food. Chem. 56 (19) (2008) 9190-9199, https://doi.org/10.1021/jf801742w.

[49] H. Schlichtherle-Cerny, W. Grosch, Evaluation of taste compounds of stewed beef juice, Z. Lebensmittel. Untersuchung Forschung A 207 (5) (1998) 369-376, https://doi.org/10.1007/s002170050347.

[50] Y. Liu, C. Zhang, S. Chen, Comparison of active non-volatile taste components in the viscera and adductor muscles of oyster (Ostrearivularis Gould), Food Sci. Technol. Res. 19 (3) (2013) 417-424, https://doi.org/10.3136/fstr.19.417.

[51] A. Giri, K. Osako, T. Ohshima, Identification and characterisation of headspace volatiles of fish miso, a Japanese fish meat based fermented paste, with special emphasis on effect of fish species and meat washing, Food Chem. 120 (2) (2010) 621-631, https://doi.org/10.1016/j.foodchem.2009.10.036. 